\title{
XVIII.
}

\section{Zum Mechanismus der subcutanen Zerreissungen des Quadriceps, der Patella und des Ligamentum patellae.}

\author{
Von \\ Dr. Karl Walz \\ in Baiersbronn.
}

Ein durch seine Aetiologie interessanter Fall von Zerreissung der Quadricepssehne, den ich zu beobachten Gelegenheit hatte, gab mir Veranlassung, mich mit dem Mechanismus dieser und ähnlicher Verletzungen am Extensionsapparate des Knies zu beschäftigen.

Am 2. October 1896 wurde ich zu dem 70 jährigen Holzfäller J. G. in M. gerufen, welcher angeblich sein Bein gebrochen hatte. Derselbe war einige Stunden vorher im Wald verungltickt. Er war mit noch 2 Kameraden beschäftigt gewesen, eine sogenannte „Wulze" aufzurichten, das heisst das mit der Erde, Steinen und Moosdecke durch den Wind aus dem Boden gerissene Wurzelwerk einer grossen Tanne, welches eine mehrere Centner schwere, uibermannshohe Platte bildete. Die 3 Arbeiter hatten die Wulze nahezu auf die Kante gestellt - der Stamm und die grösseren Wurzeln waren vorher abgesägt. Plötzlich kam die Platte anf dem steilen Terrain ins Rutschen. Hierbei entglitt eine schenkeldicke, abgesägte Wurzel, an welcher G. mit aller Kraft gehoben hatte, und die sich der Mitte des gestreckten linken Oberschenkels gegenüber befand, - den Händen des Arbeiters, rutschte am Oberschenkel hinab und schlug mit der unteren scharfen Kante auf das Knie auf. G. stiirzte zu Boden und konnte das linke Bein nicht mehr bewegen.

Der Befund war charakteristisch: Die Haut des linken Oberschenkels war vollkommen unverletzt, Schwellung bestand keine. Es fiel sofort eine rinnenförmige Einziehung der Haut dicht tiber der Patella ins Auge. In die Rinne konnte man gerade einen Finger querlegen. Der obere Rand der Patella und noch über denselben hinaus ein kleiner Theil der Gelenkfläche derselben war leicht abzutasten. Sie war sehr leicht nach unten und den Seiten zu bewegen. Ihre Längenmaasse waren genau die gleichen wie rechts. $1 \frac{1 / 2}{\mathrm{Cm}}$. oberhalb der Kniescheibe fühlte man deutlich die scharfe Kante der quergetrennten Quadricepssehne. Knochensplitter an derselben waren nicht zu fühlen. Wenn man die Kniescheibe mit der einen, die Sehne mit der anderen Hand fasste, konnte man durch 
seitliche entgegengesetzte Verschiebung den Aussenrand der Patella mit dem Innenrand der Sehne nahezu in eine Linie bringen und umgekehrt. Actives Strecken des gebogenen Knies war unmöglich. Bei dem Versuche, das Knie activ durchzudrücken, sah man an der Innenseite des Gelenkes einen dünnen Strang sich anspannen, die Patella hob sich jedoch nicht.

Da der Patient bei seinem Alter von einer Operation nichts wissen wollte und sich durch Heftpflasterzug, Einbinden des Oberschenkels von oben nach unten und Elevation des Beines in gestreckter Stellung eine Annäherung der zerrissenen Theile erreichen liess, wurde diese Methode eingeschlagen und mit Massage verbunden.

Ein Erguss ins Gelenk kam nicht zu Stande, nur in der Umgebung der Rissstelle entstand mässige Schwellung, so dass nach einigen Tagen die Verhältnisse nicht mehr so deutlich durchgetastet werden konnten.

Nach $4 \frac{1}{2}$ Wochen begann sich eine geringe Festigkeit an der Rissstelle zu zeigen. Die Patella folgte dem Zuge des Quadriceps nach oben. G. konnte einige Secunden das gestreckte Bein erheben. Wegen der Gefahren des langen Liegens für den alten Mann und beginnenden Aufliegens wurde daher ein Wasserglasverband angelegt, nnd konnte G. einige Tage später die ersten Gehversuche anstellen. Nach 10 Wochen war die Vereinigung fest. G. konnte mit einer Kniekappe gehen.

Bemerkenswerth ist bei dem Ort der Verletzung, dass keinerlei Bluterguss ins Gelenk stattfand, also kein Zusammenhang mit dem Gelenk vorhanden war. Als Ursache der Zerreissung wird man in der Hauptsache die schneidende Wirkung der Kante annehmen mïssen. Berücksichtigt man jedoch, dass die Richtung der schneidenden Gewalteinwirkung nicht senkrecht und quer zu den Fasern der Sehne verlief, sondern entlang derselben, so wird man ausser der schneidenden Wirkung noch ein weiteres Moment annehmen müssen. Vergegenwärtigen wir uns die Situation: Der Mann steht mit gestrecktem Bein, der Quadriceps ist durch die Anstrengung des Hebens aufs Aeusserste contrahirt, plötzlich fällt ein schwerer, kantiger Gegenstand mit Wucht am Oberschenkel hinunter und muss an dem nur wenig vorspringenden, oberen Patellarrand einen Widerstand finden. Durch die Quadricepscontraction wird die Patella nach oben gezogen, das kantige Wurzelende reisst die Kniescheibe nach unten. Irgendwo muss eine Ruptur erfolgen. Vermuthlich hat durch schneidende Wirkung eine Kerreissung der vorderen Sehnenpartie stattgefunden, durch die nach oben und unten reissende Gewalt wird die nun geschwächte Sebne vollends zerrissen.

Schwieriger ist die Erklärung in Fällen, bei denen es sich um reinen Muskelzug ohne äussere Gewalteinwirkung handelt. Wunseh 1 ) citirt den Ausspruch Bähr's: „Warum reisst nun bei dieser indireeten

1) Deutsche Zeitschrift f'. Chirurgie. Bd. XLIII. S. 617. 
Gewalteinwirkung bald die Patella, bald das Ligamentum, bald aber auch die Tuberositas tibiae ab? Auf diese Fragen geben die einschlägigen Abhandlungen keine bestimmte Auskunft." Ich werde im Folgenden versuchen, auf Grund physikalischer Gesetze und der mechanischen Verbältnisse des Kniegelenkes eine Erklärung zu finden.

Die ausführlichste Arbeit über diesen Gegenstand hat May d ${ }^{1}$ ) im Jahre 1882 veröffentlicht und die einschlägigen Fälle aus der Literatur zusammengestellt. Er bringt 49 Fälle von Zerreissung der Sehne, 12 von Muskelriss, 63 von Ligamentruptur zusammen. Berger hat im Dictionnaire encyclopédique des sciences médicales 1877 48 Fälle von Ligamentruptur aufgeftihrt. Hafemann ${ }^{2}$ ) hat 64 Fälle von Sehnenruptur aufzuweisen. Weitere Fälle von Sehnenruptur be-

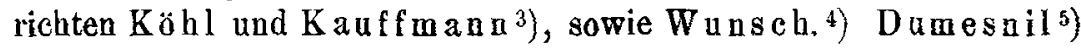
ffigt 5 Fälle von unvollständiger Ligamentruptur hinzu.

Die Brïche der Patella sind demnach weit häufiger. Sie bilden 1,3 Proc. aller Knochenbrüche; MaydI berechnet nach einigen Statistiken, dass mindestens 30 Proc. aller Patellarbrüche durch Muskelzug entstehen.

Unter den 67 Fällen der Sehnenzerreissung von Maydl, Hafemann, Köbl, Kauffmann und Wunsch war die Rissstelle $12 \mathrm{mal}$ im Verlauf der Sehne, 13 mal dicht über der Patella, 25 mal an der Insertionsstelle an der Patella, 16 mal ohne nähere Bezeichnung.

Von den 63 Fällen der Ligamentruptur bei Maydl ist $26 \mathrm{mal}$ die Rissstelle angegeben, nämlich $3 \mathrm{mal}$ genau an der Patella, $1 \mathrm{mal}$ ebenso mit Abriss von Knochenstüekehen, 6 mal in der oberen Hälfte, $3 \mathrm{mal}$ ungefähr in der Mitte, $13 \mathrm{mal}$ an der Tibialinsertion, hiervon 4 mal mit Abriss der Tuberositas.

Es ist somit nicht ganzrichtig, dass die Quadriceps. sehne sehrviel seltener als das Ligament zerreisst, wie allgemein in den Lehrbüchern angenommen wird, sondern beides dürte etwa gleich oft vorkommen.

In Maydl's und Hafemann's Fällen fand 11 mal doppelseitige Sehnenruptur statt, 3 mal zerriss zuerst die rechte, später auch die linke Sehne. Dagegen sind nur 2 Fälle von gleichzeitiger doppelter Ligamentruptur bekannt.

1) Deutsche Zeitschrift f. Chirurgie. Bd. XVII u. XVIII.

2) Ueber subcutane Zerreissung des M. ext. cruris quadriceps. Dissertation. Beriin 1885.

3) Correspondenzblatt fur Schweizer Aerzte. 1888 u. 1893.

4) Deutsche Zeitschrift f. Chirurgie, Bd. XLIII. S. 617.

5) Contribution à l'étude des, ruptures incomplètes du ligament rotulien. Thèse. Paris 1889. 
Zum.Mechanismus der subcutanen Zerreissungen des Quadriceps u. s. w. 433

Das Maximum der Patellarfracturen ist nach Maydl im 30. bis 50. Lebensjahr, das der Sebnen- und Ligamentruptur im 40.-60.

Von 57 Fällen von Sehnenruptur waren 51 Männer und 6 Frauen betroffen. Von 45 Ligamentrupturen kamen 40 auf das männliche Geschlecht.

Maydl sucht nachzuweisen, dass sich Muskel, Sehnen und Knochen bei den Rupturen ganz in derselben Weise verhalten, wenigstens in der Mehrzahl der Fälle; mit dem Unterschied, dass Sehne und Knochen immer passiv zerrissen werden, eine Muskelruptur durch eigene Thätigkeit immerhin zugestanden werden, aber eine plausible Erklärung für sie noch gesucht werden müsse. Er stellt die verschiedenen Ansichten zusammen, wonach

$A$. die Zerreissung durch Muskelaction erfolgen kann.

Der Muskel in Action kann sein

I. entweder derselbe der $a$ ) sich selbst, seine Sehne oder Knocheninsertion zerreisst: Volkmann, Lossen, Pitha u. A. b) die sich contrahirenden Muskeln zerreissen ihren ebenfalls sich kontrahirenden Antagonisten, Roulin und Rust. c) Passive Dehnung des contrahirten Muskels durch die Kraft des Körpergewichtes oder eine andere fremde Gewalt mit Ausschluss der Thätigkeit der Antagonisten, besonders durch $\mathrm{M}$ ay $\mathrm{dl}$ vertreten. $d$ ) Isolirte Contraction zur Erklärung der partiellen Rupturen, Sédillot. Nach Régeard wird eine noch nicht contrahirte Muskelpartie durch eine contrahirte gedebnt.

II. Zerreissung des erschlafften Muskels durch seine Antagonisten.

$B$. Die Zerreissung erfolgt durch alleinige Aussengewalt: Druck, Schlag, Stoss und Dehnung.

C. Spiralige Drehung, wofür ein Fall Maydl's spricht.

$D$. Krämpfe, convulsive Contractionen, die dem Willen nicht mehr unterthan sind, zerreissen den Muskel.

$E$. In den anderen Fällen sei sowohl eine Contractiondes betroffenen Muskels als die Einwirkung einer jener Contraction entgegenwirkenden Kraft nachzuweisen.

In den meisten Fällen trat nach Maydl die Zerreissung bei einer Schutzbewegung gegen drohenden Fall ein. Und zwar handele es sich dann immer um einen Fall nach hinten. Auch in drei Fällen Maydl's, bei welcher ein Stürzen nach vorn angegeben ist, erfolge ein instinktives Zurückwerfen des Körpers, was dann einem Fall nach hinten gleichkommt. Ebenso ist der Vorgang beim Hängenbleiben mit einem Fuss. Beim Ausgleiten, Fallen nach rückwärts werde durch das Beraubtwerden einer Stiutze das blitzschnelle Uebertragen des Körpergewichtes auf das eine feststehende Bein erfordert. In allen 
Fällen werde eine mächtige Quadricepscontraction meist instinktiv hervorgebracht, welche jedoch selbst in ihrer böchsten Stärke nicht ausreiche, um den Oberschenkel sammt Rumpf gegen den Unterschenkel zu strecken. Das Gewicht des fallenden Körpers - vermehrt durch die allenfalls getragenen Gegenstände oder die Schnelligkeit, mit der sich der Körper im Moment des Falles fortbewege - zerreisse den gespannten Muskel, seine Sehne oder Knocheninsertion.

Warum aber bald das eine, bald das andere reisst, dariiber sagt Maydl nichts. Wunseh findet auf Grund seines und Köhl's Fall wenigstens für gewisse Arten von Sehnenrupturen die anatomische Erklärung in eingelagerten Knochengebilden, Ich glaube, dass auch bei ganz normalen Verhältnissen der Grund in rein physikalischen Momenten zu suchen ist.

Lossen ${ }^{1}$ ) sagt bei Besprechung der Patellarfractur durch Muskelzug: „Die Beugung des Knies scheint entschieden die Trennung zu begünstigen. In dieser Stellung ruht nämlich die Patella mit ibrer Mitte auf der Femurrolle auf, während der untere und obere Rand hohl liegen und durch das Ligamentum patellae einerseits, durch die Quadricepssehne andererseits im Winkel gezogen werden. Die Patella befindet sich in der Lage eines Stabes, der iber dem Knie gebrochen werden soll, und reisst in der Quere, seltener schräg ein."

Ich möchte dies ein wenig präciser so ausdrücken: Bei gebengtem Knie bildet die Patella einen zweiarmigen Hebel. Knüpfen wir an dem einfachen Bilde des tiber dem Knie zu brechenden Stabes an, und machen wir folgende einfache Versuche. Wenn wir einen Bindfaden nehmen, den wir eben noch zerreissen können, so wird es ziemlich auf dasselbe herauskommen, ob wir sie in gerader Richtung oder winklig uber dem Knie oder einer nicht scharfen Kante zerreissen. Knüpen wir dagegen zwei Stiicke derselben Schnur an die Enden eines Stäbchens, etwa eines Bleistiftes, so ist einlenchtend, dass wir bei Zug in gerader Richtung an den Schnurenden unmöglich den Bleistift zerreissen können, sondern höchstens die Schnur; ziehen wir aber im Winkel an dem über eine Kante gelegten Bleistift, so ist dieser mit leichter Mühe zu brechen. Es folgt daraus, dass es durchaus nicht gleichgiltig ist wenn in die Quadricepssehne ein fester Körper eingelagert ist, und dass auch bei weit grösserer Zugfestigkeit des Partellarknochens gegeniiber dem Sehnengewebe dennoch durch Hebelwirkung eher der Knochen

1) Verletzungen der unteren Extremitäten. 1880. S. 148. 
Zum Mechanismus der subcutanen Zerreissungen des Quadriceps u. s. w. 435

brechen muss als die Sebne, gebeugte Kniestellung und annäherud gleich lange Hebelarme vorausgesetzt.

Die Contraction des Quadriceps wirkt auf die Patella nach zwei Richtungen, als rein ziehende, zerreissende Kraft bei gestrecktem Knie; als ziehende und zugleich biegende bei gebeugtem Knie. Je stärker die Beugung des Knies ist, um so geringer wird der Zug, um so grösser die Biegung, welche ibr Maximum erreichen würde, wenn Sehne und Ligament senkrecht zur Patella ziehen wurden. Es kommt somit an der Patella im Wesentlichen die absolute oder Tensionsfestigkeit und die relative oder Biegungsfestigkeit in Betracht.

Weder uber die Tensionsfestigkeit der spongiösen Knochen, noch über die Biegungselasticität liegen genaue Daten vor, eben so wenig wie über die Festigkeit der Quadricepssebne und des Ligaments. Wir können annehmen, dass die Tensionsfestigkeit wie bei der compacten Substanz eine weit höhere ist.

Die zum Abbrechen eines Körpers nöthige Kraft steht nach bekannten Gesetzen im geraden Verhältniss zur Breite desselben und zum Quadrat seiner Höhe, verbält sich dagegen umgekebrt wie seine Länge. Daraus ergeben sich für die Patella Verschiedenheiten bei jugendlichen und bei erwachsenen Individuen. Verstärkt wird die Patella dadurch, dass ihre Gelenkfläche nicht eine Ebene, sondern zwei im Winkel zusammenstossende Flächen bildet.

Die Biegung erfolgt auf den beiden Condylen als Hypomochlion, also um eine quer durch das Gelenk gehende Axe. Allein es ist doch nicht ganz zu iubersehen, dass namentlich bei gestrecktem Knie eine wenn auch sehr geringe Biegung um eine schräge Axe zu Stande kommt. Lossen ${ }^{1}$ ) sagt bei Besprechung der Luxationen der Patella: „Für das so sehr viel häufigere Auftreten der Luxationen nach aussen wird allgemein die geringere Höhe des Condylus externus, sowie die physiologische Valgusstellung des Knies verantwortlich gemacht. Es kann nicht geleugnet werden, dass schon bei dem normalen Kniegelenke die Resultante aller Quadricepsfasern in einem nach aussen offenen Winkel auf den Unterschenkel wirkt. Wäre die Patella nicht in ihrer Rinne festgehalten, so müsste sie bei jeder Streckbewegung, dem Muskelzuge folgend, ein wenig nach aussen rücken. In böherem Grade wäre dies bei dem pathologisehen Genu valgum der Fall." Ich glaube, dass sich durch diesen Umstand manche Schrägbrüche der Kniescheibe erklären lassen.

Wir haben bei der Biegung der Patella bisber angenommen,

1) 1. c. S. 142. 
dass die Patella mit ihrer Mitte auf den Condylen aufliegt, und ihre beiden Hälften zwei gleich lange Hebelarme bilden. Wenn wir wieder auf unseren einfachen Versuch mit dem an zwei Schnürchen gebundenen Stäbchen zurückgreifen, so erreichen wir durch Verlängerung des einen Hebelarmes bald einen Punkt, wo nicht mehr der Stab zerbricht, sondern der am kurzeren Hebelarm befestigte Bindfaden reisst, wenn derselbe mit seinem freien Ende auch befestigt ist. Dieser Punkt wird um so näher der Mitte des Stabes liegen, je kürzer dieser ist. Die Kräfte verhalten sich umgekehrt wie ihre Hebelarme. So können geringe individuelle Verschiedenheiten im Ban der Patella die Kraftwirkung zu Gunsten der Quad. ricepssehne oder des Ligamentes verändern. Auch hier ist die Kraftwirkung um so grösser, je gebeugter das Knie ist, je mehr sich die Zugrichtung an der Patella der Senkrechten nähert. Berücksichtigt muss dabei werden, dass die Richtung des Zuges der Sehne derjenigen des Ligamentes nicht ganz gleich ist. Bei rechtwinklig gebeugtem Koie beträgt der erstere Winkel nach einigen von mir ausgeführten Messungen durchsehnittlich 500, der letztere $40^{\circ}$. Für das Ligament ist daher die Kraftwirkung ungunstiger. Ferner muss bei dem reinen Zuge am gebeugten Knie Kraft dadurch verloren gehen, dass die Patella an die Condylen angepresst wird, und dadurch eine Reibung entsteht.

Man kann die in Betracht kommenden Verletzungen in drei Abtheilungen ordnen, in solche, die durch äussere Gewalt allein entstanden sind, solehe, bei denen der Muskelzug allein die Ursache ist, und ferner diejenigen, bei welchen Beides in Betracht kommt, Unter den Fracturen kommen, wie erwähnt, etwa $30 \%$ durch Muskelzug zu Stande. Unter 48 Fällen von Ligamentruptur nach Berger ${ }^{1}$ ) war nur einmal directe Gewalt angegeben, unter den 63 Fällen Maydl's nur 2 mal. Von 64 Fällen der Sehnenruptur nach Maydl und $\mathrm{Hafemann}$ wird $7 \mathrm{mal}$ directe Gewalt, weitere $4 \mathrm{mal}$ verbunden mit Muskelzug beschuldigt. Meist handelt es sich dabei um einen Fall aufs Knie. Die Sehnenruptur trat nach $\mathrm{Hafemann}$ 4 mal durch Fallen gegen einen kantigen Gegenstand ein. Den erwähnten Fall $W$ anseh erklärt der Referent der Aerztlichen Sachverständigenzeitung ${ }^{2}$ ) auf dieselbe Weise. Doch ist dagegen anzu. führen, dass nur bei seitlichem Fall gegen eine Kante dieser Vorgang denkbar ist. Bei einem Fall von oben nach unten ist schwer
1) Dumesnil, l. c. S. 17 .
2) 1896. Nr. 19. 
zu ersehen, wie die über dem Knie befindliche Sehne mit der Kante in Berührung kommen soll. Man müsste gerade einen Fall mit nach hinten gestreckten Beinen annehmen. Eher ist jener Gefahr das Ligament ausgesetzt. Doch in dem einzigen klaren Fall Maydl's war ein über das Knie gehendes Wagenrad die Ursache. Fälle von Hieb mit kantigen Gegenständen ohne Hauttrennung sind keine mitgeteilt, wenn gleich leicht denkbar. Naturgemäss wird durch Fall auf das Knie am bäufigsten die Fractur der Patella eintreten, wie denn viele derartige Fälle berichtet werden, wenn gleich es meist schwer zu entscheiden ist, ob die Fractur schon vor dem Fall oder erst durch den Fall aufs Knie zu Stande kam.

Es sind noch weitere Ursachen der Sehnen- und Ligamentruptur denkbar, nämlich ein gewaltsamer Zug an der Patella durch äussere Gewalt nach unten oder oben. Nach Maydl ${ }^{1}$ ) hat schon Malgaigne auseinander gesetzt, „dass, wenn die Beugung im Knie bedeutend ist, die Patella vom Boden entfernt bleibt; dass sie diesen mit ihrer Spitze berihrt, wenn sich der Oberschenkel wieder zu einem rechten Winkel auf den Unterschenkel erhebt, und dass es gerade die möglichst geringe Biegung ist, in welcher die Kniescheibe mit ihrer vordern Fläche auf den Boden stösst."

Malgaigne schliesst daraus, dass die Patellarfracturen gerade bei wenig gebeugtem Knie entstehen. Ich möchte, wenn die Spitze der Patella bei rechtwinklig gebeugtem Knie auf dem Boden aufstösst, daraus auf die Möglichkeit schliessen, dass hierdurch bei einem Fall aufs Knie die Patella nach oben gerissen wird und das Ligamentum patellae durchreisst. Umgekehrt kann, wie bei unserer Beobachtung durch den Fall eines kantigen Gegenstandes am Oberschenkel hinunter die Patella nach unten gerissen werden. Dann reisst, begünstigt durch den Zug des contrahirten Muskels dieser oder seine Sehne ab.

Häufig ist die Frage, ob directe Gewalt oder Muskelzug zu beschuldigen ist, nicht zu entscheiden, da die Ruptur vorausgegangen, und der Fall erst die Folge sein kann; oder kann Beides zusammenwirken. Die zerreissende Gewalt kann auch zwar im Individuum, aber ausserhalb des Quadriceps ihren Sitz haben. Maydl nimmt, wie wir sahen, als weitaus häufigstes an, dass durch das Gewicht des nach hinten fallenden Körpers der contrahirte Muskel oder seine Sehne im weiteren Sinn zerrissen wird. Dagegen möchte ich Folgendes einwenden: Ein Vorgang dieser Art ist dann denkbar, wenn der

1) J. c. Bd. XVII. S. 540. 
betroffene oder beide Unterschenkel fest auf dem Boden stehen und nun, ohne die Stellung des Unterschenkels zum Boden zu verändern, der Körper nach hinten fällt, wobei der Winkel zwischen Unterund Oberschenkel stetig verringert, das Knie immer mehr gebeugt wird. Dies düfte aber doch selten der Fall sein. Durch die Bewegung des Rumpfes nach hinten wird eben auch der auf dem Boden stehende Unterschenkel mit seinem oberen Theil nach hinten gezogen, der Fuss plantarflectirt und dadurch einer übermässigen Flexion im Knie Einhalt gethan, in dem eben der Rücken schon den Boden beruhrt, ehe das Knie so kräftig gebeugt wird, dass der contrahirte Extensor tuberwunden wird. Zudem ist die Anfangsgeschwindigkeit des nach hinten fallenden Körpers, die durch den Widerstand des Extensors noch verringert wird, keine so grosse, dass der Muskel nicht längst Zeit hätte, in der Contraction nachzulassen, denn es handelt sich nicht um eine blitzartige, sondern mehr weniger langsame Dehnung bei diesem Vorgang. Ich glaube vielmehr, dass meist eine vehemente Muskelcontraction die alleinige Ursache ist, und glaube, dass das Gewicht des nach hinten fallenden Körpers nur die Veranlassung zu einer solchen vehementen Contraction ist. Die ubergrosse Arbeitsaufgabe löst reflectorisch eine so starke Contraction aus, wie sie durch den Willen gar nicht möglich ist.

Sehen wir doch beim Hintenuiberfallen noch manche andere Muskelrupturen eintreten, die sich nicht alle durch Zerreissung des contrahirten Muskels durch das Körpergewicht erklären lassen. So ein Riss des Rectus abdominis nach Ridherand und ein ähnlicher nach Bonnes, ein Psoasriss nach Sagot ${ }^{1}$ ). Naturgemäss sind Rupturen beim Fallen am häufigsten an dem Streckapparat der Beine, da diese durch ihre Beweglichkeit in erster Linie zur Wiederherstellung des Gleichgewichtes und zur Wiederaufrichtung des Körpers geschaffen sind.

Ich halte es also nicht für möglich, dass eine willkürliche Muskelcontraction allein eine Ruptur verursachen kann. Eine solche ist nur denkbar, wenn sich der Contraction ein plötzlicher, unvermuteter Widerstand entgegensetzt, oder wenn die Contraction krampfartig, reflectorisch ist. Fälle von Zerreissung der Patella durch Convulsionen sind mehrfach berichtet.

Die Ruptur durch Muskelaction findet an der Stelle statt, welche entweder pathologisch oder physiologisch die schwächste ist. Krankhafte locale Veränderungen, namentlich bedingt durch vorausgegangene

1) Maydi, l. c. Bd. XVII. S. 329 u. 354. 
Verletzungen werden vielfach beschuldigt. Maydl') hält gegenüber anderen älteren Ansichten dafür, dass es nicht die Texturveränderung der Knochen, Sehnen, Ligamente ist, sondern die durch jene Verletzungen oder Erkrankungen bedingte temporäre oder dauernde Verminderung der Brauchbarkeit eines Kniegelenkes (sei es durch Schmerzen, Steifigkeit, Ankylose u. s. w.), welche jene Folgeverletzụngen herbei führt, indem der Ausfall eines wichtigen, in allen drei Categorien stets gleichen Factors (d. h. der ungehinderten Benutzung des einen Kniegelenkes) schon bei den wenigen, gewöhnlichen Verwendungsarten unserer Unterextremitäten zu typischen Unfällen (Fall auf die Knie, Fall rïcklings, mit dem Versuche, sich aufrecht zu erhalten) führt, durch die Verletzungen veranlasst werden, welche wieder gemeinschaftlich jenen typischen Mechanismus aufweisen (directe Gewalt und erfolgreiche Ueberwindung einer Muskelaction durch verschiedene Kräfte)."

Für einen pathologischen Zustand sprechen wobl die nicht seltenen Fälle, in welchen zuerst die eine Patella brach und später die andere, ähnliche Beispiele finden sich auch für Sehne und Ligament. Als prädisponirend für Muskelruptur fand sich in einem Fall Maydl's fettige Degeneration. In zwei Fällen bei Hafemann trat Sehnenruptur bei Tabes ohne jede Muskelaction ein. Du mesnil nimmt als prädisponirend für Ligamentruptur ,gichtische Diathese“ an. Wunsch führt seinen und Köhl's Fall - Ruptur der Sehne, in der sich Knorpel eingelagert fanden - auf einen chronischen Entzïndungszustand im Kniegelenk zurück. Es lässt sich denken, dass Knochennatrophien, wie sie bei Marasmus, Senilität, Inactivität, Neurosen, Syphilis auftreten, eine Fractur begunstigen. Auffallend ist dagegen, dass Patellarfracturen gerade im Alter so selten sind. Vielleicht hängt dies damit zusammen, dass ältere Personen vorsichtig gehen, wie M ayd l die Seltenheit ähnlicher Verletzungen bei Frauen darauf zurückführt, dass diese mehr zu Haus bleiben.

In den meisten Fällen ist ein pathologischer Zustand des Extensionsapparates nicht nachzuweisen, und reisst dieser am physiologisch schwächsten Punkt. Wir müssen unterscheiden zwischen Verletzungen bei gestrecktem und gebeugtem Knie. Leider ist der Beugezustand des Knies selten angegeben. Daher ist es nicht möglich, für jeden concreten Fall den Mechanismus zu beweisen. Was geschieht nun bei einer ïbermässigen Muskelcontraction, wenn das Knie gestreckt ist? Allgemein wird anerkannt, dass die Luxationen der Patella

1) 1. c. Bd. XVIII. S. 4 i. 
meist bei gestrecktem oder nahezu gestrecktem Knie vorkommen, in welcher Stellung die Gelenkkapsel erschlafft, die Patella am beweglichsten ist. $\left.\mathrm{K} \ddot{\mathrm{n}} \mathrm{ig}^{1}\right)$ sagt: „Bei vielen Fällen ist eine Muskelcontraction mitwirkend, wenn nicht alleiu maassgebend. Während z. B. der Fuss nach aussen, dass Knie nach innen gewendet ist, wie beim Ringen, thut der Verletzte einen Fehltritt, das Knie knickt nach innen um, der Unterschenkel dreht sich nach aussen, und eine jetzt eintretende starke Contraction der Extensoren, welche das Zubodenfallen hindern soll, ist im Stande, die Kniescheibe auf die äussere Fläche des äusseren Schenkelknorrens herumzuschleudern (S tre ubel)“. Allgemein wird der Grund neben solcher Einknickung in der erwähnten, nach innen schiefen Richtung im Zug der Extensorsehne gesucht. Wie die Patellarfracturen, so scheinen auch die Sehnen- und Ligamentrupturen bei gestrecktem Knie selten zu sein. In einem Falle M a ydl's, der unaufgeklärt ist, rissen bei einem ruhig auf der Treppe stehenden Mann plötzlich beide Quadricepssehnen durch. In zwei weiteren Fällen war mit der Sehnenruptur gleichzeitig Luxation der Patella nach aussen verbunden. Maydl sagt ${ }^{2}$ ): „selbstverständlich betrachten wir die Luxation als consecutiv." War das Bein gestreckt, so ist doch die Möglichkeit vorhanden, dass durch die Muskelcontraction die Luxation erfolgte, und dann erst die Sehne riss. Dieselbe Einwärtsdrehung des Knies, welche neben der schiefen Zugrichtung des Muskels die Luxation der Patella beguinstigt, vermebrt auch die über dem Condylus externus biegende Wirkung des Muskelzuges. Trotz feblender genauer Zahlen nehme ich an, dass durch Muskelzug bei gestrecktem Knie in erster Linie die Luxation oder, wenn die Kniescheibe nicht über den Condylus externus tritt, die Patellarfractur, selten die Zerreissung der sehnigen Theile erfolgt.

Bei gebeugtem Knie ist eine Luxation der fest zwischen die Condylen gepressten Patella nahezu ausgeschlossen. Hierbei ist weitaus das häufigste bei heftiger Muskelcontraction die Fractur, und zwar, wie wir oben sahen, nicht wegen geringeren Zusammenhaltens des Knochengewebes gegenüber der Sehne, sondern infolge Hebelwirkung. Wenn die Patella bei jugendlichen Individuen so selten bricht, glaube ich dies auf die Küre der Hebelarme zurückführen zu müssen. Dass die Patella nicht mit einer Fläche, sondern nur in geringer Zone den Condylen aufliegt, davon kann man sich leicht uberzengen, indem bei gestrecktem Bein und schlaffer Musculatur sie

1) Lehrbuch der spec. Chirurgie. 1889. S. 390.

2) l. c. Bd. XVII. S. 530 . 
sich wiegenartig um eine quere Axe drehen lässt. $O b$ die Berührungspunkte mit den Condylen, wenn die Kniescheibe fixirt ist, oberhalb oder unterhalb der Mitte ist, darüber fehlen Angaben, doch lässt sich denken, dass darin individuelle Schwankungen unterlaufen, und dass die Hebelarme bei ein und demselben Individuum bei verschiedenen Bewegungen und Kniestellungen nicht immer gleich lang sind. Es ist doch wahrscheinlich, dass die Patella nicht immer wieder mit den gleichen Punkten die Condylen berührt, und dass ein Unterschied vorhanden ist, ob die Contraction in gestreckter Stellung erfolgt und dann erst das Knie gebeugt wird, oder ob die Contraction erst nach vollzogener Beugung eintritt; durch Zug am oberen Ende der Patella bei gebeugtem Knie wird das obere Ende dem Femur genähert, das untere entfernt, woraus eine Verkürzung des Hebelarmes für die Quadricepssehne resultirte. Der Ort des Kniescheibenbruches ist bekanntlich bald in der Mitte, bald in der oberen oder unteren Hälfte, er hängt eben mit der Länge der Hebelarme zusammen. An einem gewissen Punkt wird jedoch der längere Hebelarm nicht mehr im Stande sein, die Kniescheibe zu brechen, sondern das am kürzeren Hebelarm befestigte Sehnengebilde zerreisst. Wir haben gesehen, dass bei gebeugtem Knie sowohl durch die Reibung der Patella, als durch die Annäberung des oberen Kniescheibenendes gegen den Femur die Verbältnisse eher für die Quadricepsruptur güstig sind. Günstig für diese muss auch folgender Umstand wirken, der gleichfalls nirgends Beachtung fand: Je mehr das Knie gebeugt ist, um so mehr vergrössert sich der Winkel zwischen Kniescheibe und Sehne. Es muss daher am Ansatz von Knochen und Sehne die obere (vordere) Hälfte der Sehne gedehnt, die untere (hintere) comprimirt werden. Dies mag erklären, dass gerade an dieser Stelle die Sehnenruptur am häufigsten ist. Aehnliche Verhältnisse, doch, wegen kleineren Winkels, nicht in gleich bohem Maasse, finden sich am Ligament. Wenn trotzdem das Ligament am häufigsten an der Tuberositas und nicht an der Patellarinsertion abreisst, so liegt wohl die Ursache darin, dass das Ligament am unteren Patellarrand eine breitere Insertionsfläche findet. Wenn in drei Fällen Maydl's auf eine Patellarfractur später eine Ligamentruptur folgte, so erkläre ich dies durch den Ausfall der Hebelwirkung bei der nicht knöchern verheilten Kniescheibe.

Es eribrigt noch kurt, auf die Muskelruptur zu sprechen zu kommen. Bei keinem Fall der Literatur lässt sich eine Zerreissung des erschlafften Mnskels nachweisen, stets handelt es sich um einen Muskel in Action. Es liegt nahe, die Ruptur mit den langsamer 
verlaufenden Rupturen glatter Muskelfasern zu vergleichen, die ja am Uterus genau studirt sind. Wie dort das untere Uterussegment gedehnt wird, so muss auch bei einem am Ursprung und Ende befestigten Muskel bei der Contraction am oberen und unteren Ende eine Debnung erfolgen, wenn der Befestigungpunkt nicht nachgiebt. Dass eine ungleichzeitige Muskelcontraction gerade am Oberschenkel ermöglicht ist, ist durch die Zusammensetzung des Extensors aus vier Köpfen erklärt. Ein vorzeitig contrahirter Muskel genügt entfernt nicht, die Last zu überwinden, genügt aber auch nicht, die Patella zu brechen oder gar die Sebne zu zerreissen und zerreisst selbst. Ich möchte dabei noch auf einen Umstand aufmerksam machen, dass beim Hintenïberfallen, wenn man nach Maydl eine Zerreissung des contrahirten Muskels durch das Körpergewicht annimmt, noch eine isolirte Dehnung des Rectus stattfinden kann, nähmlich wenn gleichzeitig mit der Beugung im Knie eine Streckung im Huftgelenk vor sich geht. Der Grund liegt in der Insertion des Rectus an der Spina inferior.

Ich möchte demnach hinsichtlich des Mechanismus folgende Einteilung der Verletzungen aufstellen, welche durch Muskelzug, bei nicht patbologischen Verhältnissen und ohne äussere Krafteinwirkung am Extensionsapparat des Knies entstehen:

I. Bei ungleichzeitigem Muskelzug entsteht die Muskelruptur.

II. Bei gleichzeitiger Muskelcontraction entsteht:

$A$. bei gestrecktem Knie am häufigsten die Luxation und, theils durch einen Zug, theils durch Biegung um den Condylus externus die Fractura patellae, selten, theilweise durch Hebelwirkung zerreissen die sehnigen Theile.

$B$. bei gebeugtem Knie am häufigsten die Fractura patellae infolge Hebelwirkung; seltener, je nach Veränderung der Länge der Hebelarme, die Zerreissung der Sehne oder des Ligamentes. 